\title{
ON BADLY APPROXIMABLE NUMBERS AND CERTAIN GAMES
}

\author{
BY \\ WOLFGANG M. SCHMIDT
}

1. Introduction. A number $\alpha$ is called badly approximable if $|\alpha-p / q|>c / q^{2}$ for some $c>0$ and all rationals $p / q$. It is known that an irrational number $\alpha$ is badly approximable if and only if the partial denominators in its continued fraction are bounded [4, Theorem 23]. In a recent paper [7] I proved results of the following type: If $f_{1}, f_{2}, \cdots$ are differentiable functions whose derivatives are continuous and vanish nowhere, then there are continuum-many numbers $\alpha$ such that all the numbers $f_{1}(\alpha), f_{2}(\alpha), \cdots$ are badly approximable.

Let $0<\alpha<1 / 2,0<\beta<1$, and consider the following game of two players black and white. First black picks a closed interval $B_{1}$. Then white picks a closed interval $W_{1} \subset B_{1}$ whose length is $\alpha$ times the length of $B_{1}$. Then black chooses an interval $B_{2} \subset W_{1}$ which is closed and has length $\beta$ times the length of $W_{1}$. Then again white picks a closed interval $W_{2} \subset B_{2}$ of length $\alpha$ times the length of $B_{2}$, and so on. Call white the winner of a play if the intersection of the intervals $W_{J}$ is badly approximable; otherwise black is called the winner.

Who will win? Since the badly approximable numbers have Lebesgue measure zero, [4, Theorem 29], one might think that black can always win. It turns out, however, that white can always win (Theorem 3).

We shall show that sets $S$ with this property (namely that white can always play such that the intersection $\bigcap W_{j}$ is in $S$ ) necessarily contain continuum-many elements (Lemma 23), that countable intersections of sets with this property again have this property (Theorem 2), and that if $S$ has this property, and $f(x)$ has a continuous derivative with $f^{\prime}(x) \neq 0$ everywhere, then the set of $\alpha$ with $f(\alpha) \in S$ again has this property (Theorem 1 ). These facts imply the result stated at the beginning.

We shall discuss games of much greater generality than those mentioned in this introduction.

The author is indebted to the referee for very valuable suggestions.

2. (F, (5)-games. Let $M, \Omega$ be sets, $\Omega^{\prime}$ a subset of $\Omega$, and $\alpha$ a mapping from $\Omega$ into subsets of $M$. Call $\mathfrak{h}$-function any function $\mathfrak{H}$ which assigns to every $B \in \Omega$ a nonempty set $\mathfrak{S}(B) \subset \Omega$ such that for $C \in \mathfrak{H}(B)$,

$$
\alpha(C) \subset \alpha(B) \text {. }
$$

Received by the editors April 30, 1965. 
Now let $\mathfrak{F}, \mathfrak{G}$ be $\mathfrak{S}$-functions, and let $S$ be an arbitrary subset of $M$. Consider the following game of two players black and white. First black picks an element $B_{1} \in \Omega^{\prime}$. Then white picks $W_{1} \in \mathfrak{F}\left(B_{1}\right)$. Then black picks $B_{2} \in \mathfrak{G}\left(W_{1}\right)$. Then again white chooses an element $W_{2} \in \mathfrak{F}\left(B_{2}\right)$, and so forth. Put $B_{i}^{*}=\alpha\left(B_{i}\right), W_{i}^{*}=\alpha\left(W_{i}\right)$ $(i=1,2, \cdots)$. One has $B_{1}^{*} \supset W_{1}^{*} \supset B_{2}^{*} \supset W_{2}^{*} \supset \cdots$. We call white the winner of a play if $\bigcap_{i=1}^{\infty} W_{i}^{*}=\bigcap_{i=1}^{\infty} B_{i}^{*}$ is contained in $S$; otherwise black is the winner. This game we call $(\mathfrak{F}, \mathfrak{G} ; S)$-game. We call $S$ an $(\mathfrak{F}, \mathfrak{G})$-winning set if white can always win the $(\mathfrak{F}, \mathfrak{F} ; S)$-game.

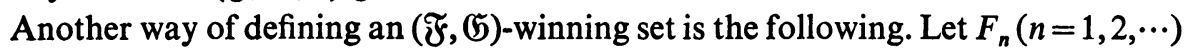
be the set of functions $f\left(B_{1}, B_{2}, \cdots, B_{n}\right)$ defined for elements $B_{l} \in \Omega$ such that $f\left(B_{1}, \cdots, B_{n}\right) \in \mathfrak{F}\left(B_{n}\right)$. A sequence $f_{1}, f_{2}, \cdots$ where $f_{n} \in F_{n}(n=1,2, \cdots)$ will be called a strategy. A strategy is called $(\mathfrak{F}, \mathfrak{G} ; S)$-winning strategy if the following holds: Let $B_{1}, B_{2}, \cdots, W_{1}, W_{2}, \cdots$ be sets such that $B_{1} \in \Omega^{\prime}$ and

$$
\begin{array}{ll}
B_{n} \in \mathfrak{G}\left(W_{n-1}\right) & (n=2,3, \cdots), \\
W_{n}=f_{n}\left(B_{1}, \cdots, B_{n}\right) & (n=1,2, \cdots) .
\end{array}
$$

Then $\bigcap_{n=1}^{\infty} B_{n}^{*}$ is necessarily contained in $S$. Now $S$ is an (F, (F))-winning set if and only if there is an $(\mathfrak{F}, \mathfrak{G} ; S)$-winning strategy. White will win by choosing $W_{n}=f_{n}\left(B_{1}, \cdots, B_{n}\right)$ when $B_{1}, \cdots, B_{n}$ are given $(n=1,2, \cdots)$.

This means that white bases his decision on how to pick $W_{n}$ not only on $B_{n}$, but also on the previous elements $B_{1}, \cdots, B_{n-1}$. Is this necessary? In other words, if $S$ is a winning set, does there exist a winning strategy of the type $f_{n}\left(B_{1}, \cdots, B_{n}\right)$ $=f\left(B_{n}\right)(n=1,2, \cdots)$, where $f \in F_{1}$ ? Such a strategy we shall call positional strategy. Using the well-ordering principle we shall show in the last section that a winning set does indeed have a positional winning strategy. This result has the following interpretation. In our game both players know the outcomes of all the previous moves. One obtains a different game if one specifies that white at the $n$th move shall know $B_{n}$ but shall not remember the number $n$ or the previous elements $B_{1}, \cdots, B_{n-1}$. Now the result on positional strategies means that if $S$ is a winning set of the original game, it is also a winning set of the new game. A special case of this was proved in $[2, \S 4]$.

Let $f_{1}, f_{2}, \cdots$ be a winning strategy. We call $B_{1}, B_{2}, \cdots$ with $B_{1} \in \Omega^{\prime}, B_{i} \in \Omega$ an $f_{1}, f_{2}, \cdots$-chain if there are elements $W_{1}, W_{2}, \cdots$ such that (1) and (2) hold. The intersection of the sets $B_{n}^{*}=\alpha\left(B_{n}\right)$ of a chain is in $S$. We call $B_{1}, \cdots, B_{k}$ a finite $f_{1}, f_{2}, \cdots$-chain if there are $B_{k+1}, B_{k+2}, \cdots$ such that $B_{1}, B_{2}, \cdots, B_{k}, B_{k+1}, \cdots$ is an $f_{1}, f_{2}, \cdots$-chain.

LeMma 1. Let $B_{1}, B_{2}, \cdots$ be elements of $\Omega$ such that $B_{1}, \cdots, B_{k}$ is a finite $f_{1}$, $f_{2}, \cdots$-chain for every $k$. Then $B_{1}, B_{2}, \cdots$ is an $f_{1}, f_{2}, \cdots$-chain.

Proof. Put $W_{n}=f_{n}\left(B_{1}, \cdots, B_{n}\right)(n=1,2, \cdots)$. Since $B_{1}, \cdots, B_{k}$ is a chain,

$$
B_{j} \in \mathbb{G}\left(W_{j-1}\right) \quad(1 \leqq j \leqq k) .
$$


Since $k$ is arbitrary, (3) holds in fact for every $j$. Hence (1) and (2) hold, and $B_{1}, B_{2}, \cdots$ is an $f_{1}, f_{2}, \cdots$-chain.

We shall repeatedly use this lemma without mention.

It is reasonable to call $S$ a losing set if black can always win. It was shown by Gale and Stewart [3] that an $(\mathfrak{F},(\mathfrak{G} ; S)$-game need not be determinate, and hence in general there are sets which are neither $(\mathfrak{F},(\mathfrak{H})$-winning nor $(\mathfrak{F},(\mathfrak{G})$-losing.

3. $(\alpha, \beta)$ - and $\left(a^{*} b\right)$-games. Already we have to specialize.

For completeness we mention Oxtoby's game [6]. Here $M$ is a topological space, and $\Omega=\Omega^{\prime}$ consists of a class of subsets with nonempty interiors such that given a nonempty open set $O$ there is a set $C \subset O, C \in \Omega$. $\alpha$ is the identity map. $\mathscr{F}(B)=\mathfrak{G}(B)$ consists of all subsets of $B$ which are in $\Omega$. A special case is the Banach-Mazurgame [5], [8]. Here $M$ is the real line and $\Omega$ consists of $M$ and all closed intervals.

Let $M$ be a complete metrical space with distance function $d(x, y)$. Let $\Omega=\Omega^{\prime}$ consist of all pairs $(\rho, c)$, where $\rho$ is a positive real number and $c \in M$. The elements $B=(\rho, c)$ of $\Omega$ will be called balls or more precisely balls of $M$, and we call $\rho=\rho(B)$ the radius, $c=c(B)$ the center of $B$. With $B \in \Omega$ we associate the set $B^{*}=\alpha(B)$ consisting of all points $x \in M$ satisfying $d(x, c) \leqq \rho$. In general, $\alpha(B)$ does not determine $B$, but it does if $M$ is a Banach space of positive dimension.

We say a ball $B_{1}=\left(\rho_{1}, c_{1}\right)$ is contained in a ball $B_{2}=\left(\rho_{2}, c_{2}\right)$ and write $B_{1} \subset B_{2}$ if

$$
\rho_{1}+d\left(c_{1}, c_{2}\right) \leqq \rho_{2} .
$$

This implies (but is not implied by) $\alpha\left(B_{1}\right) \subset \alpha\left(B_{2}\right)$. One easily checks that $B_{1} \subset B_{2}$ and $B_{2} \subset B_{3}$ implies $B_{1} \subset B_{3}$.

Let $0<\gamma<1$. Given $B \in \Omega$, let $B^{\gamma}$ be the set of all balls $B^{\prime} \subset B$ having $\rho\left(B^{\prime}\right)=\gamma \rho(B)$. Let $\mathfrak{F}^{\gamma}$ be the $\mathfrak{H}$-function defined by $\mathfrak{F}^{\gamma}(B)=B^{\gamma}$.

Now let $0<\alpha<1,0<\beta<1, S \subset M$. The $\left(\mathfrak{F}^{\alpha}, \mathfrak{F}^{\beta} ; S\right)$-game is well defined. For brevity we will call this the $(\alpha, \beta ; S)$-game. Thus in an $(\alpha, \beta)$-game, black first picks a ball $B_{1}$, then white picks a ball $W_{1} \in B_{1}^{\alpha}$, then black a ball $B_{2} \in W_{1}^{\beta}$, and so forth.

Next let $M$ be the real line and $\Omega=\Omega^{\prime}$ the set of all closed intervals of positive length. For $\alpha$ take the identity mapping. If $I$ is a closed interval and $c>1$ an integer, write ${ }^{c} I$ for the unique set of $c$ closed intervals whose lengths are $c^{-1}$ times that of $I$, and which cover $I$. Let ${ }^{c} \mathfrak{F}$ be the $\mathfrak{S}$-function with ${ }^{c} \mathfrak{F}(I)={ }^{c} I$.

Now let $a>1, b>1$ be integers, $S \subset M$. The ( $\left.{ }^{a} \mathfrak{F}{ }^{b} \mathfrak{F} ; S\right)$-game is well defined. For convenience we call it $\left(a^{*} b ; S\right)$-game.

A variant of the $\left(a^{*} a\right)$-game is the a-digit-game. Here $\Omega^{\prime}$ consists of a single element only, namely the unit-interval $0 \leqq x \leqq 1$. This game amounts to the following. First white chooses a digit $c_{1}$ to base $a$, namely $c_{1}=0,1, \cdots, a-2$ or $a-1$. Then black chooses a digit $c_{2}$, and so on. White is the winner if $x=0, c_{1} c_{2} \cdots$ (written in the scale of $a$ ) is in $S$. Every $\left(a^{*} a\right)$-winning set is an $a$-digit-winning set. 
LEMMA 2. Let $0<\alpha<1,0<\beta<1, a>1, b>1$, where $a, b$ are integers and where

$$
a b \alpha \beta=1, \quad a \alpha \geqq 2 .
$$

Then every $(a, \beta)$-winning set on the real line is $\left(a^{*} b\right)$-winning.

Proof. Here $\Omega=\Omega^{\prime}$ consists of closed intervals of positive length, and $\alpha$ is the identity map. Thus we need not distinguish between $B \in \Omega$ and the set $B^{*}=\alpha(B) \subset M$. Let $h_{1}, h_{2}, \cdots$ be an $(\alpha, \beta ; S)$-winning strategy. Given $B_{1}, \cdots, B_{n}$, put $\tilde{W}_{n}=h_{n}\left(B_{1}, \cdots, B_{n}\right)$. The length $l\left(\tilde{W}_{n}\right)$ of $\tilde{W}_{n}$ satisfies $l\left(\tilde{W}_{n}\right)=\alpha l\left(B_{n}\right) \geqq 2 a^{-1} l\left(B_{n}\right)$. Hence there is a $W_{n} \in^{a} B_{n}, W_{n} \subset \tilde{W}_{n}$. Put $f_{n}\left(B_{1}, \cdots, B_{n}\right)=W_{n}$. We claim $f_{1}, f_{2}, \cdots$ to be an $\left(a^{*} b ; S\right)$-winning strategy. Suppose (1), (2) hold with $\mathfrak{G}={ }^{b} \mathfrak{F}$. Then

$$
\begin{array}{rlrl}
B_{n} & \in \tilde{W}_{n-1}^{\beta} & (n=2,3, \cdots), \\
\tilde{W}_{n}=h_{n}\left(B_{1}, \cdots, B_{n}\right) & (n=1,2, \cdots)
\end{array}
$$

hold. (4) is true because $B_{n} \subset W_{n-1} \subset \tilde{W}_{n-1}, B_{n} \in{ }^{b} W_{n-1}, W_{n-1} \in \tilde{W}_{n-1}^{(\alpha a)^{-1}}$, and $(\alpha a b)^{-1}=\beta$. By (4), (5), $B_{1}, B_{2}, \cdots$ is a $h_{1}, h_{2}, \cdots$-chain of the $(\alpha, \beta ; S)$-game, and $\bigcap B_{n}$ is in $S$.

LEMMA 3. Let $0<\alpha<1,0<\beta<1, a>1, b>1$,

$$
a b \alpha \beta=1, \quad b \beta \geqq 2
$$

where $a, b$ are integers. Then every $\left(a^{*} b\right)$-winning set is also $(\alpha, \beta)$-winning.

Proof. Let $h_{1}, h_{2}, \cdots$ be an $\left(a^{*} b ; S\right)$-winning strategy. Define $f_{n}$ by induction on $n$ as follows. Given a closed interval $B_{1}$ pick some $\widetilde{B}_{1} \in B_{1}^{(b \beta)^{-1}}$, then $W_{1}=h_{1}\left(\widetilde{B}_{1}\right)$. Define $f_{1}\left(B_{1}\right)=W_{1}$. Given $B_{1}, \cdots, B_{n}, n>1$, put $W_{n-1}=f_{n-1}\left(B_{1}, \cdots, B_{n-1}\right)$, and pick $\widetilde{B}_{n}$ such that $\widetilde{B}_{n} \subset B_{n}, \widetilde{B}_{n} \in{ }^{b} W_{n-1}$. This is possible since $l\left(B_{n}\right)=\beta l\left(W_{n-1}\right)$ $\geqq 2 b^{-1} l\left(W_{n-1}\right)$. (Here we used $B_{n} \subset W_{n-1}^{\beta}$. If this is not the case, the sequence $B_{1}, B_{2}, \cdots, B_{n}$ will not occur in a play, and $f_{n}\left(B_{1}, \cdots, B_{n}\right)$ can be defined arbitrarily.) Now put $f_{n}\left(B_{1}, \cdots, B_{n}\right)=h_{n}\left(\widetilde{B}_{1}, \cdots, \widetilde{B}_{n}\right)$. We claim $f_{1}, f_{2}, \cdots$ is an $(\alpha, \beta ; S)$-winning strategy. Suppose (1), (2) hold with $\left(\mathfrak{G}=\mathfrak{F}^{\beta}\right.$. Then

$$
\begin{array}{ll}
\widetilde{B}_{n} \in{ }^{b} W_{n-1} & (n=2,3, \cdots), \\
W_{n}=h_{n}\left(\widetilde{B}_{1}, \cdots, \widetilde{B}_{n}\right) & (n=1,2, \cdots) .
\end{array}
$$

Hence $\widetilde{B}_{1}, \widetilde{B}_{2}, \cdots$ is a $h_{1}, h_{2}, \cdots$-chain for the $\left(a^{*} b\right)$-game. $\bigcap \widetilde{B}_{n}$ is in $S$ and $\bigcap W_{n}$ is in $S$.

4. More about $(\alpha, \beta)$-winning sets. Again let $\Omega$ be the set of "balls" $B=(\rho, c)$, where $\rho>0$ and $c \in M$. Given a ball $B$ of center $c$ and radius $\rho$ and a point $x \in M$ write

$$
e(x, B)=d(x, c) \rho^{-1} \text {. }
$$

One has $e(x, B)=0$ if and only if $x=c, e(x, B) \leqq 1$ if and only if $x \in \alpha(B)$. 
LEMMA 4. Let $e=e(x, B) \leqq 1$ and $0<\gamma<1$. Every ball $B^{\prime} \in B^{\gamma}$ has $e^{\prime}=e\left(x, B^{\prime}\right)$ in the interval

$$
\max \left(0,(e+\gamma-1) \gamma^{-1}\right) \leqq e^{\prime} \leqq(e+1-\gamma) \gamma^{-1}
$$

If $\gamma \leqq 1-e$, there is always a ball $B^{\prime} \in B^{\gamma}$ having $e^{\prime}=e\left(x, B^{\prime}\right)=0$. Moreover, if $M$ is a Banachspace of positive dimension with distance $d(y, z)=|y-z|$, then for every $e^{\prime}$ in the interval (6) there is a $B^{\prime} \in B^{\gamma}$ with $e\left(x, B^{\prime}\right)=e^{\prime}$.

Proof. Let $B$ have center $c$ and radius $\rho, B^{\prime} \in B^{\gamma}$ center $c^{\prime}$ and radius $\rho^{\prime}=\rho \gamma$.

$$
\begin{aligned}
e^{\prime} & =d\left(x, c^{\prime}\right) \rho^{\prime-1} \leqq\left(d(x, c)+d\left(c, c^{\prime}\right)\right) \rho^{\prime-1} \leqq\left(d(x, c)+\rho-\rho^{\prime}\right) \rho^{\prime-1} \\
& =\left(d(x, c) \rho^{-1}+1\right) \rho \rho^{\prime-1}-1=(e+1) \gamma^{-1}-1=(e+1-\gamma) \gamma^{-1}, \\
e^{\prime} & \geqq\left(d(x, c)-d\left(c, c^{\prime}\right)\right) \rho^{\prime-1} \geqq\left(d(x, c)-\rho+\rho^{\prime}\right) \rho^{\prime-1}=(e+\gamma-1) \gamma^{-1} .
\end{aligned}
$$

Hence (6) always holds.

Now let $\gamma \leqq 1-e$. The ball $B^{\prime}$ with center $x$ and radius $\gamma \rho$ is in $B$, since $\gamma \rho+d(x, c)=\gamma \rho+e \rho \leqq \rho$. Here $e^{\prime}=e\left(x, B^{\prime}\right)=0$. Next, let $M$ be a Banachspace and $\gamma>1-e$. Let $B^{\prime}$ be the ball with center $c^{\prime}=c-e^{-1}(\gamma-1)(x-c)$ and radius $\rho^{\prime}=\gamma \rho$. Now $\gamma \rho+d\left(c^{\prime}, c\right)=\gamma \rho+d(x, c)(1-\gamma) e^{-1}=\gamma \rho+e \rho(1-\gamma) e^{-1}=\rho$, hence $B^{\prime} \subset B^{\gamma}$. Furthermore, $e^{\prime}=e\left(x, B^{\prime}\right)=d\left(x, c^{\prime}\right) \rho^{\prime-1}=d(x, c)\left(1+(\gamma-1) e^{-1}\right)(\gamma \rho)^{-1}$ $=e \rho\left(1+(\gamma-1) e^{-1}\right)(\gamma \rho)^{-1}=(e+\gamma-1) \gamma^{-1}$. Thus if $M$ is a Banachspace, there is always a $B^{\prime} \subset B^{\gamma}$ whose $e^{\prime}=e\left(x, B^{\prime}\right)$ is the left endpoint of the interval (6).

Let $e>0$, put $c^{\prime}=c+e^{-1}(\gamma-1)(x-c)$ and let $B^{\prime}$ be the ball with center $c^{\prime}$ and radius $\rho^{\prime}=\rho \gamma . d\left(c, c^{\prime}\right)=d(x, c)(1-\gamma) e^{-1}=(1-\gamma) \rho=\rho-\rho^{\prime}$, and therefore $B^{\prime} \in B^{\gamma}$. Also $e^{\prime}=e\left(x, B^{\prime}\right)=d\left(x, c^{\prime}\right) \rho^{\prime-1}=d(x, c)\left(1-(\gamma-1) e^{-1}\right)(\gamma \rho)^{-1}$ $=e \rho\left(1-(\gamma-1) e^{-1}\right) \gamma^{-1} \rho^{-1}=(e+1-\gamma) \gamma^{-1}$.

If $e=0$, let $c^{\prime}$ be any point having $d\left(c, c^{\prime}\right)=\rho-\rho^{\prime}=(1-\gamma) \rho$, and let $B^{\prime}$ be the ball with center $c^{\prime}$ and radius $\rho^{\prime}=\gamma \rho$. Then $B^{\prime} \subset B^{\gamma}$ and $e^{\prime}=e\left(x, B^{\prime}\right)$ $=d\left(x, c^{\prime}\right) \rho^{\prime-1}=d\left(c, c^{\prime}\right) \rho^{\prime-1}=(1-\gamma) \rho \gamma^{-1} \rho^{-1}=(1-\gamma) \gamma^{-1}$.

Hence if $M$ is a Banachspace there is always a ball $B^{\prime} \in B^{\gamma}$ whose $e^{\prime}=e\left(x, B^{\prime}\right)$ equals the right endpoint of the interval (6). Since for $B^{\prime} \subset B^{\gamma}, e^{\prime}=e\left(x, B^{\prime}\right)$ depends continuously on the center $c^{\prime}$ of $B^{\prime}$, there is a ball $B^{\prime} \subset B^{\gamma}$ whose $e\left(x, B^{\prime}\right)$ equals $e^{\prime}$, where $e^{\prime}$ is an arbitrary number in the interval (6).

Lemma 5. Suppose $0<\alpha<1,0<\beta<1,2 \alpha \geqq 1+\alpha \beta$. Then the only $(\alpha, \beta)$ winning set is $M$ itself.

Proof. Let $x \in M$. Black may choose $B_{1}$ with center $x$. Hence $e_{1}=e_{1}\left(x, B_{1}\right)=0$. Then $W_{1} \in B_{1}^{\alpha}$ satisfies $e_{1}^{\prime}=e\left(x, W_{1}\right) \leqq(1-\alpha) \alpha^{-1}$ by Lemma 4. Now $\beta \leqq \beta+(2 \alpha-1-\alpha \beta) \alpha^{-1}=2-\alpha^{-1}=1-(1-\alpha) \alpha^{-1} \leqq 1-e_{1}^{\prime}$, hence by Lemma 4 black can choose $B_{2} \in W_{1}^{\beta}$ with $e_{2}=e\left(x, B_{2}\right)=0$. Thus $B_{2}$ also has center $x$. In this fashion black can enforce that $x$ is the center of every ball $B_{n}$. 
Then $x$ is in the intersection of the "ballsets" $\alpha\left(B_{n}\right)=B_{n}^{*}$ and every winning set $S$ must contain $x$. Since $x$ was arbitrary, $S=M$.

Lemma 6. Let $0<\alpha<1,0<\beta<1,2 \beta \geqq 1+\alpha \beta$. Then every dense set $S$ is $(\alpha, \beta)$-winning.

Proof. Let $S$ be dense, and suppose black picks a ball with center $c$ and radius $\rho$. There is an $x \in S$ having $d(x, c) \leqq(1-\alpha) \rho$. White may pick $W_{1} \subset B_{1}^{\alpha}$ with center $x$. Now, using the same method black used in Lemma 5 , white can enforce that all the balls $W_{n}$ have center $x$.

LEMma 7. Let $M$ be a Banachspace of positive dimension, $0<\alpha<1,0<\beta<1$, $2 \alpha<1+\alpha \beta$. Then any set $R$ obtained by removing a finite number of points from a winning set $S$ is again a winning set.

Proof. Let $R$ be obtained from the winning set $S$ by removing $x$. If black picks $B_{1}$ such that $x \notin B_{1}^{*}$, then of course white can win. If in fact at some stage of a play there occurs a $B_{n}$ with $x \notin B_{n}^{*}$, then white can win. Hence it suffices to show that white can play in such a way that $x \notin B_{n}^{*}$ for some $n$.

Assume $x \in B_{1}^{*}$ and set $e_{1}=e\left(x, B_{1}\right) \leqq 1$. White can pick a ball $W_{1}$ having $e_{1}^{\prime}=e\left(x, W_{1}\right)\left(e_{1}+1-\alpha\right) \alpha^{-1}$ by Lemma 4 . If $e_{1}^{\prime}>1, x \notin W_{1}^{*}$, and we are through. Otherwise $e_{1}^{\prime}+\beta-1=\left(e_{1}+1-\alpha\right) \alpha^{-1}+\beta-1=e_{1} \alpha^{-1}+(1+\alpha \beta-2 \alpha) \alpha^{-1}>0$ and $B_{2} \in W_{1}^{\beta}$ satisfies $e_{2}=e\left(x, B_{2}\right) \geqq e_{1} \alpha^{-1} \beta^{-1}+(1+\alpha \beta-2 \alpha)(\alpha \beta)^{-1}>e_{1}(\alpha \beta)^{-1}$ by Lemma 4 .

Generally, if $x \in B_{n}^{*}$, white can play such that either $x \notin W_{n}^{*}$ or

$$
e\left(x, B_{n+1}\right)>(\alpha \beta)^{-1} e\left(x, B_{n}\right) \text {. }
$$

Since $(\alpha \beta)^{-1}>1$, there will sooner or later occur a ball $B_{m}$ with $x \notin B_{m}^{*}$.

Lemma 8. Let $0<\alpha<1,0<\beta<1,0<\alpha^{\prime}<1,0<\beta^{\prime}<1, \alpha \beta=\alpha^{\prime} \beta^{\prime}, \alpha^{\prime} \leqq \alpha$. Then every $(\alpha, \beta)$-winning set is also $\left(\alpha^{\prime}, \beta^{\prime}\right)$-winning.

Proof. Assume $\alpha^{\prime}<\alpha$. Let $h_{1}, h_{2}, \cdots$ be an $(\alpha, \beta ; S)$-winning strategy. Given $B_{1}, \cdots, B_{n}$, write $\tilde{W}_{n}=h_{n}\left(B_{1}, \cdots, B_{n}\right)$, pick some $W_{n} \in \tilde{W}_{n}^{\alpha^{\prime} / \alpha}$ and put $f_{n}\left(B_{1}, \cdots, B_{n}\right)=W_{n}$. Suppose (1), (2) hold with $\left(\mathfrak{S}=\mathfrak{F}^{\beta^{\prime}}\right.$. Then

$$
\begin{aligned}
B_{n} \in \tilde{W}_{n-1}^{\beta^{\prime} \alpha^{\prime} / \alpha} & =\tilde{W}_{n-1}^{\beta} & & (n=2,3, \cdots), \\
\tilde{W}_{n} & =h_{n}\left(B_{1}, \cdots, B_{n}\right) & & (n=1,2, \cdots) .
\end{aligned}
$$

Hence $B_{1}, B_{2}, \cdots$ is a $h_{1}, h_{2}, \cdots$-chain of the $(\alpha, \beta)$-game and $\bigcap B_{n}^{*}$ is in $S$. Therefore $f_{1}, f_{2}, \cdots$ is an $\left(\alpha^{\prime}, \beta^{\prime} ; S\right)$-winning strategy.

LEMMA 9. Every $(\alpha, \beta)$-winning set is $\left(\alpha(\beta \alpha)^{k}, \beta\right)$-winning for $k=0,1,2, \cdots$.

Proof. Suppose in the $(\alpha, \beta)$-game, white not only makes his choices of the balls $W_{n}$, but also of the balls $B_{n}$, except those where $(k+1) \mid(n-1)$ (that is, $k+1$ 
divides $n-1)$. Thus black can pick only every $(k+1)$ st ball $B_{n}$, namely $B_{1}, B_{1+(k+1)}, B_{1+2(k+1)}, \cdots$. The balls

$$
B_{1}, W_{k+1}, B_{1+(k+1)}, W_{2(k+1)}, B_{1+2(k+1)}, \cdots
$$

are balls of an $\left(\alpha(\beta \alpha)^{k}, \beta\right)$-play. If white can win the $(\alpha, \beta)$-game it certainly can win the $\left(\alpha(\beta \alpha)^{k}, \beta\right)$-game.

CoROllaRY. Let $\alpha^{\prime} \beta^{\prime}=(\alpha \beta)^{k}$ for some integer $k>0$ and $\beta^{\prime} \geqq \beta$. Then every $(\alpha, \beta)$-winning set is $\left(\alpha^{\prime}, \beta^{\prime}\right)$-winning.

Proof. Combine Lemmas 8 and 9.

Problem. Is it true that an $(\alpha, \beta)$-winning set is necessarily $\left(\alpha^{\prime}, \beta^{\prime}\right)$-winning if $\alpha^{\prime} \leqq \alpha, \beta^{\prime} \geqq \beta$ ? In particular is this true if $M$ is the real line?

5. Behavior of winning sets under local isometries. Let $M, M^{\prime}$ be metrical spaces with distance-functions $d(x, y)$ and $d^{\prime}\left(x^{\prime}, y^{\prime}\right)$, respectively. Assume that for every ball $B$ of $M, \alpha(B)=B^{*}$ is compact, and make the same assumption on $M^{\prime} . M, M^{\prime}$ are then locally compact and complete. Let $\sigma$ be a homeomorphism from $M$ onto $M^{\prime}$. The function

$$
\bar{\mu}(x, y)=d^{\prime}(\sigma(x), \sigma(y)) / d(x, y)
$$

is defined and continuous for $x \neq y$. We call $\sigma$ a local isometry if $\bar{\mu}$ can be continued to a function $\mu$ which is defined, continuous and $\neq 0$ for all $x, y$ in $M$.

THEOREM 1. Let $\sigma$ be a local isometry from $M$ onto $M^{\prime}$. Let $S \subset M$ be an $(\alpha, \beta)$-winning set. Then $S^{\prime}=\sigma(S) \subset M^{\prime}$ is an $\left(\alpha^{\prime}, \beta^{\prime}\right)$-winning set if $\alpha^{\prime} \beta^{\prime}=\alpha \beta, \alpha^{\prime}<\alpha$.

We first need a lemma. Write $\tau$ for the inverse map of $\sigma$ and define $v\left(x^{\prime}, y^{\prime}\right)$ for $x^{\prime}, y^{\prime} \in M^{\prime}$ by either of the following two equivalent formulae:

$$
v\left(x^{\prime}, y^{\prime}\right)=1 / \mu\left(\tau\left(x^{\prime}\right), \tau\left(y^{\prime}\right)\right), \quad \mu(x, y)=1 / v(\sigma(x), \sigma(y)) .
$$

When $x^{\prime} \neq y^{\prime}, v\left(x^{\prime}, y^{\prime}\right)=d\left(\tau\left(x^{\prime}\right), \tau\left(y^{\prime}\right)\right) / d^{\prime}\left(x^{\prime}, y^{\prime}\right)$. Given $\lambda>0$ and a ball $B$ of $M$ with center $c$ and radius $\rho$ write $\sigma(\lambda, B)$ for the ball $B^{\prime}$ of $M^{\prime}$ with center $\sigma(c)$ and radius $\lambda \rho$. Given $\lambda>0$ and a ball $B^{\prime}$ of $M^{\prime}$ with center $c^{\prime}$ and radius $\rho^{\prime}$ write $\tau\left(\lambda, B^{\prime}\right)$ for the ball $B$ of $M$ with center $\tau\left(c^{\prime}\right)$ and radius $\lambda \rho^{\prime}$. Denote the set of balls $C^{\prime} \subset B^{\prime}$ with $\rho^{\prime}\left(C^{\prime}\right) \leqq \delta \rho^{\prime}\left(B^{\prime}\right)$ by $B^{\prime \delta-}$.

Lemma 10. Let $B^{\prime}$ be a ball of $M^{\prime}$, and $\varepsilon>0$. Put $v_{0}=\max v\left(x^{\prime}, y^{\prime}\right)$, taken over all $\left(x^{\prime}, y^{\prime}\right) \in B^{\prime *} \times B^{\prime *}$.

There exists a $\delta=\delta\left(B^{\prime}, \varepsilon\right)>0$ such that every $C^{\prime} \in B^{\prime \delta-}$ has the following property.

Put $v=v\left(c^{\prime}, c^{\prime}\right)$ and $\mu=v^{-1}=\mu\left(\tau\left(c^{\prime}\right), \tau\left(c^{\prime}\right)\right)$, where $c^{\prime}$ is the center of $C^{\prime}$. Now for any ball $D^{\prime} \subset C^{\prime}$ of $M^{\prime}$ and any ball $D$ of $M$,

$$
D \subset \tau\left(\nu(1-\varepsilon), D^{\prime}\right) \text { implies } \sigma(\mu(1-\varepsilon), D) \subset D^{\prime} .
$$


On the other hand, if $E$ is a ball of $M$ with $E \subset \tau\left(v_{0}, C^{\prime}\right)$ and $E^{\prime}$ a ball of $M^{\prime}$,

$$
E^{\prime} \subset \sigma(\mu(1-\varepsilon), E) \text { implies } \tau\left(v(1-\varepsilon), E^{\prime}\right) \subset E .
$$

Proof. Let $B=\tau\left(2 v_{0}, B^{\prime}\right) . \mu(x, y)$ is uniformly continuous and bounded from below in $B^{*} \times B^{*}$. Hence there is an $\eta=\eta\left(B^{\prime}, \varepsilon\right)>0$ such that

$$
\left|\frac{\mu\left(x_{1}, y_{1}\right)}{\mu\left(x_{2}, y_{2}\right)}-1\right|<\varepsilon
$$

if $x_{1}, x_{2}, y_{1}, y_{2}$ are in $B^{*}$ and $d\left(x_{1}, x_{2}\right) \leqq \eta, d\left(y_{1}, y_{2}\right) \leqq \eta$.

Set $\delta_{1}=\eta\left(3 v_{0} \rho\left(B^{\prime}\right)\right)^{-1}$, let $C^{\prime} \in B^{\prime \delta_{1}-}$ and suppose $D, D^{\prime}$ satisfy the hypothesis of (9). Now if $D=\left(d, \rho_{d}\right), D^{\prime}=\left(d^{\prime}, \rho_{d}^{\prime}\right), B^{\prime}=\left(b^{\prime}, \rho_{b}^{\prime}\right), C^{\prime}=\left(c^{\prime}, \rho_{c}^{\prime}\right)$

$$
\rho_{d}+d\left(d, \tau\left(d^{\prime}\right)\right) \leqq v(1-\varepsilon) \rho_{d}^{\prime} .
$$

Now since $D^{\prime} \subset C^{\prime} \subset B{ }^{\prime}, d^{\prime}\left(d^{\prime}, b^{\prime}\right) \leqq \rho_{b}^{\prime}$, whence $d\left(\tau\left(d^{\prime}\right), \tau\left(b^{\prime}\right)\right) \leqq v_{0} \rho_{b}^{\prime}$. Similarly, $d\left(\tau\left(c^{\prime}\right), \tau\left(b^{\prime}\right)\right) \leqq v_{0} \rho_{b}^{\prime}$. Finally, by $(12), \quad d\left(d, \tau\left(b^{\prime}\right)\right) \leqq d\left(d, \tau\left(d^{\prime}\right)\right)+d\left(\tau\left(d^{\prime}\right), \tau\left(b^{\prime}\right)\right)$ $\leqq v \rho_{d}^{\prime}+v_{0} \rho_{b}^{\prime} \leqq 2 v_{0} \rho_{b}^{\prime}$. Since $B$ has radius $2 v_{0} \rho_{b}^{\prime}$, the points $\tau\left(d^{\prime}\right), \tau\left(c^{\prime}\right), d$ are all in $B^{*}$.

Furthermore, $d\left(\tau\left(d^{\prime}\right), \tau\left(c^{\prime}\right)\right) \leqq v_{0} d^{\prime}\left(d^{\prime}, c^{\prime}\right) \leqq v_{0} \rho_{c}^{\prime} \leqq v_{0} \delta_{1} \rho_{b}^{\prime}<\eta / 2, d\left(d, \tau\left(c^{\prime}\right)\right)$ $\leqq d\left(d, \tau\left(d^{\prime}\right)\right)+d\left(\tau\left(d^{\prime}\right), \tau\left(c^{\prime}\right)\right) \leqq v \rho_{d}^{\prime}+\eta / 2 \leqq v_{0} \delta_{1} \rho_{b}^{\prime}+\eta / 2 \leqq \eta$. Hence by (11),

$$
\mu\left(\tau\left(d^{\prime}\right), d\right)<\mu\left(\tau\left(c^{\prime}\right), \tau\left(c^{\prime}\right)\right)(1+\varepsilon)=\mu(1+\varepsilon) .
$$

Now

$$
\begin{aligned}
\mu(1-\varepsilon) \rho_{d}+d^{\prime}\left(\sigma(d), d^{\prime}\right) & <\mu \rho_{d}+\mu\left(d, \tau\left(d^{\prime}\right)\right) d\left(d, \tau\left(d^{\prime}\right)\right) \\
& <\mu(1+\varepsilon)\left(\rho_{d}+d\left(d, \tau\left(d^{\prime}\right)\right)\right. \\
& \leqq \mu v(1-\varepsilon)(1+\varepsilon) \rho_{d}^{\prime}<\rho_{d}^{\prime}
\end{aligned}
$$

Thus the conclusion of (9) holds.

Hence to obtain (9), one may take $\delta=\delta_{1}$. Now, by symmetry, one may treat $B$ as we did $B^{\prime}$. There is a $\delta_{2}>0$, such that (10) holds if $C \in B^{\delta_{2}-}, E \subset C$, and if $E^{\prime}$ is a ball of $M^{\prime}$. We set $\delta=\min \left(\delta_{1}, \delta_{2}\right)$ and show (10) holds for $E \subset \tau\left(v_{0}, C^{\prime}\right)$. By what we just said it suffices to verify $\tau\left(v_{0}, C^{\prime}\right) \in B^{\delta_{2}-}$.

We have $C^{\prime} \subset B^{\prime} \delta-$, hence $\rho_{c}^{\prime} \leqq \delta \rho_{b}^{\prime}$ and $\rho_{c}^{\prime}+d^{\prime}\left(c^{\prime}, b^{\prime}\right) \leqq \rho_{b}^{\prime}$. Since $b^{\prime}, c^{\prime}$ are in $B^{\prime *}, \quad v_{0} \rho_{c}^{\prime}+d\left(\tau\left(c^{\prime}\right), \tau\left(b^{\prime}\right)\right) \leqq v_{0} \rho_{c^{\prime}}+v_{0} d^{\prime}\left(c^{\prime}, b^{\prime}\right) \leqq v_{0} \rho_{b}^{\prime}$, whence $\tau\left(v_{0}, C^{\prime}\right) \subset B$. Finally, the radius of $\tau\left(v_{0}, C^{\prime}\right)$ is $v_{0} \rho_{c}^{\prime} \leqq \delta v_{0} \rho_{b}^{\prime}<\delta_{2} \rho_{b}$.

Proof of Theorem 1. Let the hypotheses of the theorem be satisfied. There is an $\varepsilon>0$ such that $\alpha^{\prime}=\alpha(1-\varepsilon)^{2}, \beta^{\prime}=\beta(1-\varepsilon)^{-2}$. Suppose black starts with a ball $B_{1}^{\prime}$. Choose $\delta=\delta\left(B_{1}^{\prime}, \varepsilon\right)$. Since $\alpha^{\prime} \beta^{\prime}<1$, some ball $B^{\prime}$ of the play will be in $B_{1}^{\prime}$. Let $v=v\left(b_{j}^{\prime}, b_{j}^{\prime}\right)$, where $b_{j}^{\prime}$ is the center of $B_{j}^{\prime}$, and $\mu=v^{-1}$. Then (9) will hold for balls $D^{\prime} \subset B_{j}^{\prime}$ of $M^{\prime}$ and balls $D$ of $M$, while (10) will hold for balls $E \subset \tau\left(v_{0}, B_{j}^{\prime}\right)$ 
of $M$ and $E^{\prime}$ of $M^{\prime}$, where $v_{0}=\max \left(x^{\prime}, y^{\prime}\right)$, taken over all $\left(x^{\prime}, y^{\prime}\right) \in B_{1}^{\prime *} \times B_{1}^{\prime *}$. We may assume this already to be true for $j=1$.

Let $f_{1}, f_{2}, \cdots$ be an $(\alpha, \beta ; S)$-winning strategy. We claim the functions $f_{1}^{\prime}, f_{2}^{\prime}, \cdots$ defined by

$$
f_{n}^{\prime}\left(B_{1}^{\prime}, \cdots, B_{n}^{\prime}\right)=\sigma\left(\mu(1-\varepsilon), f_{n}\left(\tau\left(v(1-\varepsilon), B_{1}^{\prime}\right), \cdots, \tau\left(v(1-\varepsilon), B_{n}^{\prime}\right)\right)\right) \quad(n=1,2, \cdots)
$$

are an $\left(\alpha^{\prime}, \beta^{\prime} ; S^{\prime}\right)$-winning strategy for plays beginning with our particular $B_{1}^{\prime}$.

First we have to verify $f_{n}^{\prime}\left(B_{1}^{\prime}, \cdots, B_{n}^{\prime}\right) \in B_{n}^{\prime \alpha^{\prime}}$. For this purpose set $B_{i}=\tau\left(v(1-\varepsilon), B_{i}^{\prime}\right)$ $(i=1, \cdots, n)$ and $W_{n}=f_{n}\left(B_{1}, \cdots, B_{n}\right)$. Now $W_{n} \subset B_{n}=\tau\left(v(1-\varepsilon), B_{n}^{\prime}\right)$, whence $f_{n}^{\prime}\left(B_{1}^{\prime}, \cdots, B_{n}^{\prime}\right)=\sigma\left(\mu(1-\varepsilon), W_{n}\right) \subset B_{n}^{\prime}$ by (9). (After all, $\left.B_{n}^{\prime} \subset B_{1}^{\prime}\right)$. A comparison of radii actually shows $f_{n}^{\prime}\left(B_{1}, \cdots, B_{n}\right) \in B_{n}^{\prime \alpha^{\prime}}$.

Now let balls $B_{1}^{\prime}, B_{2}^{\prime}, \cdots ; W_{1}^{\prime}, W_{2}^{\prime}, \cdots$ of $M^{\prime}$ satisfy

$$
\begin{array}{ll}
B_{n}^{\prime} \in W_{n-1}^{\prime \beta^{\prime}} & (n=2,3, \cdots), \\
W_{n}^{\prime}=f_{n}^{\prime}\left(B_{1}^{\prime}, \cdots, B_{n}^{\prime}\right) & (n=1,2, \cdots) .
\end{array}
$$

Put $B_{n}=\tau\left(v(1-\varepsilon), B_{n}^{\prime}\right), \quad W_{n}=f_{n}\left(B_{1}, \cdots, B_{n}\right) \quad(n=1,2, \cdots)$. One has $B_{n-1}^{\prime} \subset B_{1}^{\prime}$, hence $\rho_{b_{n-1}}^{\prime}+d^{\prime}\left(b_{1}^{\prime}, b_{n-1}^{\prime}\right) \leqq \rho_{b_{1}}^{\prime}$, whence $v_{0} \rho_{b_{n-1}}^{\prime}+d\left(\tau\left(b_{1}^{\prime}\right), \tau\left(b_{n-1}^{\prime}\right)\right) \leqq v_{0} \rho_{b_{1}}^{\prime}$, which gives $\tau\left(v_{0}, B_{n-1}^{\prime}\right) \subset \tau\left(v_{0}, B_{1}^{\prime}\right) . \quad W_{n-1} \subset B_{n-1}=\tau\left(v(1-\varepsilon), B_{n-1}^{\prime}\right) \subset \tau\left(v_{0}, B_{n-1}^{\prime}\right)$ $\subset \tau\left(v_{0}, B_{1}^{\prime}\right)$. Hence we may apply (10) with $E=W_{n-1}$ and see that $B_{n}^{\prime} \subset W_{n-1}^{\prime}$ $=f_{n-1}^{\prime}\left(B_{1}^{\prime}, \cdots, B_{n-1}^{\prime}\right)=\sigma\left(\mu(1-\varepsilon), W_{n-1}\right)$ implies

$$
B_{n}=\tau\left(v(1-\varepsilon), B_{n}^{\prime}\right) \subset W_{n-1} \quad(n=2,3, \cdots) .
$$

Using this and $W_{n}=f_{n}\left(B_{1}, \cdots, B_{n}\right)(n=1,2, \cdots)$, we conclude $x=\bigcap B_{n}^{*} \in S$.

Let $x^{\prime}=\bigcap B_{n}^{\prime *}$. This implies $d^{\prime}\left(x^{\prime}, b_{n}^{\prime}\right) \leqq \rho_{b_{n}}^{\prime}$, whence $d\left(\tau\left(x^{\prime}\right), b_{n}\right)=d\left(\tau\left(x^{\prime}\right), \tau\left(b_{n}^{\prime}\right)\right)$ $\leqq v_{0} \rho_{b_{n}}^{\prime}$. Since $b_{n}$ tends toward $x$ and $\rho_{b_{n}} \rightarrow 0, \tau\left(x^{\prime}\right)=x$, whence $x^{\prime}=\sigma(x) \in \sigma(S)=S^{\prime}$.

6. $\alpha$-winning sets. Let $0<\alpha<1$. Call a subset $S$ of a complete metrical space $\alpha$-winning, if it is $(\alpha, \beta)$-winning for every $\beta, 0<\beta<1$.

Lemma 11. Let $0<\alpha^{\prime}<\alpha<1$. Then every $\alpha$-winning set is $\alpha^{\prime}$-winning.

Proof. Given any $\beta^{\prime}, 0<\beta^{\prime}<1$, there exists a $\beta, 0<\beta<1$, such that $\alpha \beta=\alpha^{\prime} \beta^{\prime} . S$ is $(\alpha, \beta)$-winning, hence $\left(\alpha^{\prime}, \beta^{\prime}\right)$-winning by Lemma 8 .

LEMMA 12. The only $\alpha$-winning set $S \subset M$ with $\alpha>1 / 2$ is $S=M$ itself.

Proof. There is a $\beta, 0<\beta<1$, having $2 \alpha \geqq 1+\alpha \beta$. The result is now an immediate consequence of Lemma 5 .

Let $S \subset M$. Define the winning dimension of $S$,

$$
\text { windim } S \text {, }
$$

as follows. Windim $S=0$ if $S$ is $\alpha$-winning for no $\alpha>0$. Otherwise windim is the least upper bound of all $\alpha$ in $0<\alpha<1$ such that $S$ is $\alpha$-winning. It follows from Lemma 12 that windim $S=1$ if and only if $S=M$; otherwise $0 \leqq$ windim $S \leqq 1 / 2$. 
LEMMA 13. Let $M, M^{\prime}$ be metrical spaces such that for balls $B$ of $M$ and $B^{\prime}$ of $M^{\prime}, \alpha(B)$ and $\alpha\left(B^{\prime}\right)$ are compact. Let $\sigma$ be a local isometry from $M$ onto $M^{\prime}$ and let $S \subset M$. Then

$$
\text { windim } \sigma(S)=\operatorname{windim} S
$$

\section{Proof. Apply Theorem 1.}

THEOREM 2. The intersection of countably many $\alpha$-winning sets is $\alpha$-winning.

Corollary. Windim $\left(\bigcap_{j=1}^{\infty} S_{j}\right)=$ g.l.b. (windim $\left.S_{j}\right)$.

Proof of Theorem 2. We have to show that $S=\bigcap S_{j}$ is $\alpha$-winning if each of the sets $S_{j}$ is. We show that $S$ is $(\alpha, \beta)$-winning, say. White will win by playing according to the following rule.

At the first, third, fifth, $\cdots$ move, white moves according to an $\left(\alpha, \alpha \beta \alpha ; S_{1}\right)$ winning strategy. Since $B_{2 l+1} \in B_{21-1}^{\alpha \beta \alpha}$, white can enforce in this way that $\bigcap B_{n}^{*}$ is in $S_{1}$, no matter what strategy he uses in his second, fourth, sixth, $\cdots$ move.

At the second, sixth, tenth, $\cdots$ move, white uses an $\left(\alpha, \alpha(\beta \alpha)^{3} ; S_{2}\right)$-winning strategy. Generally, at the $k$ th move, where $k \equiv 2^{l-1}\left(\bmod 2^{l}\right)$, white moves as if he were playing the $\left(\alpha, \alpha(\beta \alpha)^{2^{2}-1}: S\right)$-game, and thus can enforce that $\bigcap B_{n}^{*}$ is in $S$.

Our rule amounts to this: Let $f_{1}^{l}, f_{2}^{l}, \cdots(l=1,2, \cdots)$ be an $\left(\alpha, \alpha(\alpha \beta)^{2^{l-1}} ; S_{l}\right)$ winning strategy. We now define a strategy $f_{1}, f_{2}, \cdots$ as follows. For $k \equiv 2^{l-1}\left(\bmod 2^{l}\right)$ and $k=2^{l-1}+(t-1) 2^{l}$, set

$$
f_{k}\left(B_{1}, \cdots, B_{k}^{l}\right)=f_{t}\left(B_{2^{l-1}}, B_{2^{l-1}+2^{l}}, \cdots, B_{2^{l-1}+(t-1) 2^{l}}\right) \text {. }
$$

Then

$$
\begin{array}{ll}
B_{2^{t-1}+(t-1) 2^{l}} \in W_{2^{l}-1+(t-2) 2}^{\alpha(\beta \alpha) 2^{t-1}} & (t=2,3, \cdots), \\
W_{2^{t-1}+(t-1) 2^{l}}=f_{t}^{l}\left(B_{2^{t-1}}, \cdots, B_{2^{t-1}+(t-1) 2^{l}}\right) & (t=1,2, \cdots),
\end{array}
$$

and the intersection

$$
\bigcap_{t=1}^{\infty} B_{2^{i-1}+(t-1) 2^{t}}^{*}
$$

is in $S_{l}(l=1,2, \cdots)$.

LEMMA 14. Let $M$ be a Banachspace. Let $T$ be be obtained from an $\alpha$-winning set $S, S \subset M, 0<\alpha \leqq 1 / 2$, by deleting at most countably many points. Then $T$ is also $\alpha$-winning.

Proof. Let $T$ be obtained by removing the points $x_{1}, x_{2}, \cdots$ from $S$, and let $T_{j}$ be obtained by removing $x_{1}, \cdots, x_{j}$ from $S$. Each of the sets $T_{j}$ is $\alpha$-winning by Lemma 7 , hence $T$ is $\alpha$-winning by Theorem 2 . 
7. Badly approximable numbers. In this section $M$ is the space of real numbers with the usual metric, and the badly approximable numbers are considered as subset of $M$.

From here until the end of $\S 12, \alpha$ will be $1-1$. Hence we need not and will not distinguish between elements $B \in \Omega$ and sets $B^{*}=\alpha(B) \subset M$.

THEOREM 3. The set $S$ of badly approximable numbers is $(\alpha, \beta)$-winning for every $\alpha, \beta$ having $0<\alpha<1,0<\beta<1,2 \alpha<1+\alpha \beta$.

Corollary. Windim $S=1 / 2$.

REMARK. Badly approximable numbers can be generalized to $n$-tuples, and then the analogous theorem holds. See [1] or [7].

Proposition. Let $0<\alpha<1,0<\beta<1, \gamma=1+\alpha \beta-2 \alpha>0$. Suppose black begins his play with a ball of radius $\rho$ ( = interval of length $2 \rho$ ). Put $\delta=(\gamma / 2) \min \left(\rho, \alpha^{2} \beta^{2} \gamma / 8\right)$. Then white can enforce that $x=\bigcap B_{n}$ satisfies $|x-p / q|>\delta q^{-2}$ for all integers $p$ and $q \neq 0$.

Obviously this proposition implies the theorem. Note that reals $x$ with $|x-p / q|>\delta q^{-2}$ have partial denominators $\leqq \delta^{-1}$.

Lemma 15. Let $\alpha, \beta, \gamma$ be like in the proposition. Let the integer $t$ satisfy $(\alpha \beta)^{t}<\gamma / 2$. Assume $a$ ball $B_{k}$ with center $b_{k}$ and radius $\rho_{k}$ occurs in some $(\alpha, \beta)$-play. Then white can play in such a way that $B_{k+t}$ is contained in the "halfline" $x>b_{k}+\rho_{k} \gamma / 2$.

Proof. Let $g^{+} \in F_{1}^{\alpha}$ be the function which assigns to an interval $B$ of center $c$ and length $2 \rho$ the interval with center $c+\rho(1-\alpha)$ and length $2 \alpha \rho$. Now for given $B_{k+i}, 0 \leqq i<t$, white chooses $W_{k+i}=g^{+}\left(B_{k+i}\right)$. Denote the center of $B_{n}$ by $b_{n}$, the center of $W_{n}$ by $w_{n}(n=1,2, \cdots)$. Then $w_{k}=b_{k}+\rho_{k}(1-\alpha)$, $b_{k+1} \geqq w_{k}-\alpha \rho_{k}(1-\beta) \geqq b_{k}+\rho_{k} \gamma>b_{k}$, and $b_{k+t} \geqq b_{k}+\rho_{k} \gamma$. Since $B_{k+t}$ has radius $(\alpha \beta)^{t} \rho_{k}<\rho_{k} \gamma / 2, B_{k+t}$ is in the halfline $x>b_{k}+\rho_{k} \gamma / 2$.

Proof of the Proposition. We may assume black starts with a ball $B_{1}$ of radius $\rho \leqq \alpha \beta \gamma / 8$. Otherwise, if $\rho>\alpha \beta \gamma / 8$, there will be a first $B_{j}$ in the course of the play having radius $\rho_{j} \leqq \alpha \beta \gamma / 8$, and then $\alpha^{2} \beta^{2} \gamma / 8<\rho_{j} \leqq \alpha \beta \gamma / 8$. Since both $\rho>\alpha^{2} \beta^{2} \gamma / 8$ and $\rho_{j}>\alpha^{2} \beta^{2} \gamma / 8$, it does not matter whether $\delta$ is defined using $\rho$ or $\rho_{j}$, and white can play as if $B_{j}$ were the first black ball. Hence assume

$$
\rho \leqq \alpha \beta \gamma / 8 \text {. }
$$

Choose the integer $t$ such that $\alpha \beta \gamma / 2 \leqq(\alpha \beta)^{t}<\gamma / 2$ and define $R>0$ by

$$
R^{2}(\alpha \beta)^{t}=1 \text {. }
$$

To prove the proposition it will suffice to show that white can play in such a way that 


$$
|x-p / q|>\delta q^{-2}
$$

whenever $(p, q)=1$ (that is, $p$ and $q$ are relatively prime),

$$
x \in B_{n t+1} \text { and } 0<q<R^{n}
$$

for some integer $n \geqq 0$.

Clearly (14) holds if (15) holds for $n=0$, since $0<q<R^{0}=1$ has no integral solution $q$. Suppose $B_{1}, B_{t+1}, B_{2 t+1}, \cdots, B_{(k-1) t+1}$ are already such that (14) holds if (15) holds for $0 \leqq n \leqq k-1$. Now in the next moves white has to worry only over fractions $p / q$ where $R^{k-1} \leqq q<R^{k}$. In fact white has to worry over at most one such fraction: If $|x-p / q|<\delta / q^{2},\left|x^{\prime}-p^{\prime}\right| q^{\prime} \mid<\delta / q^{\prime 2}$, where $R^{k-1} \leqq q<R^{k}$, $R^{k-1} \leqq q^{\prime}<R^{k}, p / q \neq p^{\prime} \mid q^{\prime}, x, x^{\prime}$ both in $B_{(k-1) t+1}$, then $\left|p / q-p^{\prime}\right| q^{\prime} \mid \leqq \delta / q^{2}$ $+\delta / q^{\prime 2}+2 \rho\left(B_{(k-1) t+1}\right) \leqq 2 \delta R^{2-2 k}+2 \rho(\alpha \beta)^{(k-1) t}=2(\rho+\delta) R^{2-2 k}<4 \rho R^{2-2 k}$ $\leqq \frac{1}{2} \alpha \beta \gamma R^{2-2 k}=\frac{1}{2} \alpha \beta \gamma(\alpha \beta)^{-t} R^{-2 k} \leqq R^{-2 k}$, while on the other hand $\left|p / q-p^{\prime}\right| q^{\prime} \mid$ $\geqq 1 /\left(q q^{\prime}\right)>R^{-2 k}$, which gives a contradiction.

Hence white has to worry over at most one subinterval $C$ of $B_{(k-1) t+1}$ of length $2 \rho(C) \leqq 2 \delta / q^{2} \leqq 2 \delta R^{2-2 k}$. Now if $C$ has its center to the left or on the center $b$ of $B_{(k-1) t+1}, C$ is contained in the halfline $x \leqq b+\delta R^{2-2 k}=b+\delta(\alpha \beta)^{(k-1) t}$ $=b+\delta \rho_{(k-1) t+1} / \rho \leqq b+\rho_{(k-1) t+1} \gamma / 2$, where $\rho_{(k-1) t+1}$ is the radius of $B_{(k-1) t+1}$. By Lemma 15 white can enforce that $B_{k t+1}$ is contained in $x>b+\rho_{(k-1) t+1} \gamma / 2$, and $B_{k t+1}$ has empty intersection with $C$. The reasoning is similar if the center of $C$ is to the right of the center of $B_{(k-1) t+1}$.

There is an analogy of $\alpha$-winning sets with residual sets ( $=$ complements of sets of first category) in so far, as countable intersections of residual sets are again residual sets. By definition, residual sets are sets $T$ with the property that every intersection $T \cap O$ with a nonempty open set $O$ contains a nonempty open set $O^{\prime}$, as well as countable intersections of sets with this property.

The numbers with unbounded partial denominators in their continued fraction are a residual set. This set is the intersection of the sets $T_{k}$ of numbers with at least one partial denominator $\geqq k$, and it is easy to see that every intersection of $T_{k}$ with an open interval contains an open interval.

Thus the set of numbers with unbounded partial denominators is a residual set but not a winning set, and the set of numbers with bounded partial denominators is $\alpha$-winning for $0<\alpha \leqq 1 / 2$, but is a set of first category. This is in contrast to the situation for the Banach-Mazur game [6, Theorem 1$]$.

\section{Anormal numbers.}

THEOREM 4. Let $0<\alpha<1,0<\beta<1, \gamma=1+\alpha \beta-2 \alpha>0$. Let $g$ be an integer so large that

$$
g>4(\alpha \beta \gamma)^{-1}
$$

and let $d$ be a digit in the scale of $g$, i.e., $d=0,1, \cdots, g-2$ or $g-1$. The set $S$ 
of reals $x$ in whose "decimal" expansion to scale $g$ the digit d occurs at most finite number of times is $(\alpha, \beta)$-winning.

COROLlaRY. The sets $S_{s}^{*}$ of numbers $x$ which are not normal to base $s$ is $(\alpha, \beta)$ winning, and therefore windim $S_{s}^{*}=1 / 2$.

Proof of the Corollary. Some integral power $g$ of $s$ satisfies(16). The set of numbers with only finitely many zeros in their expansion to scale $g$ is contained in the set of numbers not normal to scale $g$, which is the same as the set of numbers not normal to scale $s$. Hence this latter set is $(\alpha, \beta)$-winning by the theorem.

Proof of Theorem 4. Let black begin with the ball $B_{1}$ of radius $\rho$. Choose integers $k \geqq 1, n_{0} \geqq 1$ such that

$$
g^{-k} / 4>(\alpha \beta)^{n_{0}-1} \rho \geqq(\alpha \beta) g^{-k} / 4
$$

Define integers $n_{1}, n_{2}, \cdots$ by

$$
g^{-k-j} / 4>(\alpha \beta)^{n_{j}-1} \rho \geqq(\alpha \beta) g^{-k-j} / 4 \quad(j=1,2, \cdots) .
$$

Then, since $\alpha \beta>4 /(g \gamma)$,

$$
g^{-k-j} / 4>(\alpha \beta)^{n_{j}-1} \rho>g^{-k-j-1} / \gamma>g^{-k-j-1} / 4 \quad(j=1,2, \cdots) .
$$

Thus $n_{0}<n_{1}<n_{2}<\cdots$.

We are going to show that white can play such that every $x \in B_{n}, j \geqq 1$, has its $(k+j)$ th digit different from $d$. Let $B_{n_{j-1}}$ be given. The numbers $x$ whose $(k+j)$ th digit equals $d$ are in intervals of length $g^{-k-j}$ whose distance is $\geqq g^{1-k-j}(1-1 / g) \geqq g^{1-k-j} / 2>2(\alpha \beta)^{n_{j-1}-1} \rho=2 \rho\left(B_{n_{j-1}}\right)$. Hence white has to worry over at most one interval $C \subset B_{n_{j-1}}$ of length $\leqq g^{-k-j}$. Let us assume without loss of generality that the center of $C$ is less or equal to the center $b$ of $B_{n_{j-1}}$. Then $C$ is contained in the halfline

$$
x<b+g^{-k-j} / 2<b+(\alpha \beta)^{n_{j-1}-1} \rho \gamma / 2=b+\rho\left(B_{n_{j-1}}\right) \gamma / 2 .
$$

Put $t=n_{j}-n_{j-1}$.

$$
(\alpha \beta)^{t}=\left((\alpha \beta)^{n_{j}-1} \rho\right) /\left((\alpha \beta)^{n_{j-1}-1} \rho\right)<\left(g^{-k-j} / 4\right) /\left(g^{-k-j} / \gamma\right)=\gamma / 4
$$

and Lemma 15 applies. White can enforce $B_{n_{j}}=B_{n_{j-1}+t}$ to be in the set of $x$ having $x>b+\rho\left(B_{n_{j-1}}\right) \gamma / 2$, and hence can enforce that $C \cap B_{n_{j}}$ is empty.

9. Numbers with infinitely many zeros in their decimal.

THeOREM 5. Let $g>2$ be integral and let $S_{g}$ be the set of reals which have infinitely zeros in their "decimal" to base $g$. Then $S_{g}$ is $\alpha_{g}=\left((g-1)^{2}+1\right)^{-1}$ winning but not $\alpha$-winning for $\alpha>\alpha_{g}$. Hence

$$
\text { windim } S_{g}=\alpha_{g}=\left((g-1)^{2}+1\right)^{-1} \text {. }
$$


Let $k \geqq 1, n$ be integers. Write $I_{k}(n)$ for the interval $\left[n g^{-k}, n g^{-k}+(g-1)^{-1} g^{-k}\right]$, $K_{k}$ for the union of all intervals $I_{k}(n), n=0, \pm 1, \pm 2, \cdots$.

LEMMA 16. Let $I$ be a closed interval of length $l(I) \leqq\left(\alpha_{g}\left(g^{2}-g\right)\right)^{-1}$. There is $a k \geqq 1$ and an interval $J \in I^{\alpha_{g}}, J \subset K_{k}$.

Proof. Choose $k \geqq 1$ satisfying

$$
\left(\alpha_{g}(g-1) g^{k+1}\right)^{-1}<l(I) \leqq\left(\alpha_{g}(g-1) g^{k}\right)^{-1} .
$$

We are going to construct an interval $J^{*} \subset I \cap K_{k}$ of length $l\left(J^{*}\right) \geqq \alpha_{g} l(I)$.

$K_{k}$ consists of intervals of length $(g-1)^{-1} g^{-k}$, the complement of $K_{k}$ of intervals of length $(g-2)(g-1)^{-1} g^{-k}$. The worst situation is when the midpoint $c$ of $I$ coincides with the midpoint of one of the intervals of the complement of $K_{k}$. In this case $I$ contains all numbers $x$ in

$$
c \leqq x \leqq c+\frac{1}{2} l(I)
$$

and $K_{k}$ contains all $x$ in

$$
\begin{aligned}
c+\frac{1}{2}(g-2)(g-1)^{-1} g^{-k} & \leqq x \leqq c+\frac{1}{2}(g-2)(g-1)^{-1} g^{-k}+(g-1)^{-1} g^{-k} \\
& =c+\frac{1}{2}(g-1)^{-1} g^{1-k} .
\end{aligned}
$$

Let $J^{*}$ consist of all $x$ in

$$
c+\frac{1}{2}(g-2)(g-1)^{-1} g^{-k} \leqq x \leqq c+\min \left(\frac{1}{2} l(I), \frac{1}{2}(g-1)^{-1} g^{1-k}\right) .
$$

Obviously $J^{*} \subset I \cap K_{k}$. Furthermore,

$$
\begin{aligned}
& l\left(J^{*}\right)-\alpha_{g} l(I) \\
& =\min \left(\frac{1}{2} l(I), \frac{1}{2}(g-1)^{-1} g^{1-k}\right)-\frac{1}{2}(g-2)(g-1)^{-1} g^{-k}-\alpha_{g} l(I) \\
& =\min \left(\left(\frac{1}{2}-\alpha_{g}\right) l(I)-\frac{1}{2}(g-2)(g-1)^{-1} g^{-k},(g-1)^{-1} g^{-k}-\alpha_{g} l(I)\right) \\
& \geqq \min \left(\frac{1}{2} g(g-2) \alpha_{g} l(I)-\frac{1}{2}(g-2)(g-1)^{-1} g^{-k}, 0\right) \geqq 0
\end{aligned}
$$

by (20).

Proof of Theorem 5. Let $n g^{-k}=c_{0}+c_{1} g^{-1}+\cdots+c_{k} g^{-k}$ where $c_{0}, c_{1}, \cdots, c_{k}$ are integers, $0 \leqq c_{j} \leqq g-1(j=1, \cdots, k)$. The interval $I_{k}(n)$ now consists of all $x$ satisfying

$$
c_{0}+c_{0} g^{-1}+\cdots+c_{k} g^{-k} \leqq x \leqq c_{0}+c_{1} g^{-1}+\cdots+c_{k} g^{-k}+g^{-k-1}+g^{-k-2}+\cdots .
$$


Hence if $x$ is in the interior of $I_{k}(n)$, at least one of the digits $c_{k+1}, c_{k+2}, \cdots$ of $x$ is zero. In fact if $x$ is in a closed subset $C$ of the interior of $I_{k}(n)$, then at least one of the digits $c_{k+1}, \cdots, c_{k+m}$ of $x$ is zero, where $m=m(C)$.

We now are going to show that $S_{g}$ is $\alpha_{g}$-winning. Let $0<\beta<1$. In the $\left(\alpha_{g}, \beta\right)$ game white plays arbitrarily until a ball $B_{j i}$, with $2 \rho\left(B_{j_{1}}\right) \leqq\left(\alpha_{g}\left(g^{2}-g\right)\right)^{-1}$ occurs. Now by the lemma, white can pick $W_{j_{1}} \subset K_{k_{1}}$, say $W_{j_{1}} \subset I_{k_{1}}\left(n_{1}\right)$. At his next move white can enforce that $W_{j_{1}+1}$ is in the interior of $I_{k_{1}}\left(n_{1}\right)$. There is an $m_{1}$ such that every $x \in W_{j_{1}+1}$ has at least one of the digits $c_{k_{1}+1}, \cdots, c_{k_{1}+m_{1}}$ equal to zero. White can play arbitrarily again until $2 \rho\left(B_{j_{2}}\right) \leqq \alpha_{g}^{-1}(g-1)^{-1} g^{-k_{1}-m_{1}}$. By the lemma, white can pick $W_{j_{2}} \subset K_{k_{2}}$ for some $k_{2}$, and obviously $k_{2} \geqq k_{1}+m_{1}$. At his next move white chooses $W_{j_{2}+1}$ in the interior of some $I_{k_{2}}\left(n_{2}\right)$, and so on.

This proves the first part of the theorem.

Let $\alpha>\alpha_{g}$. Choose $m$ integral and so large that

$$
\alpha>\left(1+2(g-1) g^{3-m}\right) \alpha_{g},
$$

and let $\beta=\alpha^{-1} g^{-m}$. We are going to show that $S_{g}$ is $(\alpha, \beta)$-losing.

Black can adopt the following strategy. First he picks the ball $B_{1}$ to consist of all $x$ in

$$
g^{-1}+g^{-2} \leqq x \leqq 2 g^{-1}+g^{-3}+g^{-4}+\cdots=2 g^{-1}+g^{-2}(g-1)^{-1} .
$$

$B_{1}$ has length $g^{-1}-g^{-2}+g^{-2}(g-1)^{-1}=\alpha_{g}^{-1} g^{-2}(g-1)^{-1}$.

For $u=0,1, \cdots,(g-1)^{2}+1$ put $y_{u}=g^{-1}+g^{-2}+u(g-1)^{-1} g^{-2}$. The numbers $y_{u}$ are at distances $(g-1)^{-1} g^{-2}$, they are contained in $B_{1}$, and $y_{0}$, $y_{(g-1) 2+1}$ are the endpoints of $B_{1}$.

$W_{1}$ will have length

$$
\begin{aligned}
l\left(W_{1}\right) & =\alpha l\left(B_{1}\right)=\alpha \alpha_{g}^{-1} g^{-2}(g-1)^{-1}>g^{-2}(g-1)^{-1}\left(1+2\left(g^{\prime}-1\right) g^{3-m}\right) \\
& =g^{-2}(g-1)^{-1}+2 g^{1-m}
\end{aligned}
$$

by (21). Let $\tilde{W}_{1}$ be the closed interval of length $g^{-2}(g-1)^{-1}$ and with the same midpoint as $W_{1} . \tilde{W}_{1}$ will contain one of the points $y_{u}, 1 \leqq u \leqq(g-1)^{2}$; say $y_{u_{0}} \in \tilde{W}_{1}$. Hence $W_{1}$ will contain the interval

$$
y_{u_{0}}-g^{1-m} \leqq x \leqq y_{u_{0}}+g^{1-m} .
$$

First consider the case (a) where $g-1$ divides $u_{0}$, say $u_{0}=l(g-1)$. Now $y_{u_{0}}=g^{-1}+g^{-2}+l g^{-2}$, where $1 \leqq l \leqq g-1$. If $l<g-1, y_{u_{0}}=0,1(l+1) 000 \cdots$ when written as a decimal in scale $g$, and $y_{u_{0}}-g^{-m}=0,1 l(g-1) \cdots(g-1) 000 \cdots$, that is, $y_{u_{0}}-g^{-m}$ will have the digits $1, l$, then $m-2$ times $g-1$, then zeros. If $l=g-1, y_{u_{0}}=0.2000 \cdots$ and $y_{u_{0}}-g^{-m}=0,1(g-1) \cdots(g-1) 000 \cdots$, that is, it will have digits $1, m-1$ times $g-1$, then zeros. Hence any $x$ in the interval 
$y_{u_{0}}-g^{-m} \leqq x<y_{u_{0}}$ has its first $m$ digits different from zero. Now black picks $B_{2}$ to be the interval

$$
y_{u_{0}}-g^{-m}+g^{-m}\left(g^{-1}+g^{-2}\right) \leqq x \leqq y_{u_{0}}-g^{-m}+g^{-m}\left(2 g^{-1}+g^{-3}+g^{-4}+\cdots\right) \text {. }
$$

$B_{2}$ is contained in (22), hence in $W_{1}$, it has length

$$
l\left(B_{2}\right)=g^{-m} l\left(B_{1}\right)=\alpha^{-1} g^{-m} l\left(W_{1}\right)=\beta l\left(W_{1}\right),
$$

and every $x \in B_{2}$ has its first $m$ digits different from zero.

Next take the case (b) where $u_{0}$ is not a multiple of $g-1$, say $u_{0}=l(g-1)+r$, $1 \leqq r \leqq g-2, l \leqq g-2$. Now

$$
y_{u_{0}}=g^{-1}+g^{-2}+l g^{-2}+r g^{-2}(g-1)^{-1}=g^{-1}+(l+1) g^{-2}+r\left(g^{-3}+g^{-4}+\cdots\right),
$$

hence $y_{u_{0}}=0,1(l+1) r r r \cdots$. Put $\bar{y}=y_{u_{0}}+g^{-m}-g^{-m-1}-g^{-m-2}-\cdots$ $=y_{u_{0}}+g^{-m}-g^{-m}(g-1)^{-1} \cdot \bar{y}=0,1(l+1) r r \cdots r(r+1) 000 \cdots$, that is, $\bar{y}$ has digits $1, l+1, m-3$ times $r, r+1$, then zeros. Any $x$ in the interval $\bar{y} \leqq x<\bar{y}+g^{-m}$ has its first $m$ digits different from zero. Now black picks $B_{2}$ to consist of all $x$ satisfying

$$
\bar{y}+g^{-m}\left(g^{-1}+g^{-2}\right) \leqq x \leqq \bar{y}+g^{-m}\left(2 g^{-1}+g^{-3}+g^{-4}+\cdots\right) .
$$

$B_{2}$ is in (22) hence in $W_{1}$, its length is $\beta l\left(W_{1}\right)$, and every $x \in B_{2}$ has its first $m$ digits different from zero.

Black does not have to worry over the first $m$ digits any more. Since $B_{2}$ is congruent to $g^{-m} B_{1}$ modulo $g^{-m}$, black can apply the same strategy to ensure that the next $m$ digits of any $x \in B_{3}$ again are all different from zero. Continuing in this way black can enforce that $x=\bigcap B_{n}$ has no zeros among its digits.

10. $a^{*}$-winning sets. Let $a>1$ be integral. A set of reals is called $a^{*}$-winning if it is $\left(a^{*} b\right)$-winning for every integer $b>1$.

LEMMA 17. Let $a^{\prime} b^{\prime}=a b$, and a divides $a^{\prime}$. Then every $\left(a^{*} b\right)$-winning set is $\left(a^{\prime *} b^{\prime}\right)$-winning.

Proof. Just as for Lemma 8.

LEMMA 18. Every $\left(a^{*} b\right)$-winning set is $\left(a(b a)^{k_{*}} b\right)$-winning for every integer $k \geqq 0$.

Proof. Just as for Lemma 9.

LEMMA 19. Let $a$ be $a$ divisor of $a^{\prime}$. Then every $a^{*}$-winning set is $a^{\prime *}$-winning.

Proof. This follows from Lemma 17.

Combining Lemma 2 and Theorem 3 one finds that the set of badly approximable numbers is $a^{*}$-winning for $a \geqq 4$. A direct examination of the proof of 
Theorem 3 shows this set to be $a^{*}$-winning for every $a \geqq 2$. A similar remark applies to anormal numbers.

11. The Hausdorff dimension of winning sets. The Hausdorff dimension of a set $S$ in a metrical space $M$ is defined as follows. $S$ has Hausdorff dimension $\infty$ if for some $\eta>0 S$ cannot be covered by countably many balls of radius $<\eta$. Otherwise put $\{S, \eta\}^{\alpha}$ for the greatest lower bound (possibly $\infty$ ) of all the sums

$$
\sum_{i=1}^{\infty} \rho\left(B_{l}\right)^{\alpha}
$$

where $B_{1}, B_{2}, \cdots$ is a covering of $S$ by balls $B_{l}$ of radius $<\eta .\{S, \eta\}^{\alpha}$ is a decreasing function of $\eta$.

$$
\{S\}^{\alpha}=\lim _{\eta \rightarrow 0}\{S, \eta\}^{\alpha}
$$

(possibly $\infty$ ) is called $\alpha$-dimensional measure of $S$. Either $\{S\}^{\alpha}=\infty$ for every $\alpha$, in which case $S$ again has Hausdorff dimension $\infty$. Or there is a unique $\delta \geqq 0$ such that $\{S\}^{\alpha}=\infty$ for $\alpha<\delta$ and $\{S\}^{\alpha}=0$ for $\alpha>\delta$, and in this case one defines the Hausdorff dimension of $S$ to be $\delta$.

THEOREM 6. Let $M$ be a Hilbertspace, and let $0<\alpha<1,0<\beta<1$. Assume there are integers $t, m$ with the following property. Given $h_{1}, h_{2}, \cdots, h_{t}$ with $h_{i} \in F_{i}^{\alpha}(i=1, \cdots, t)$ and given a ball $C_{1}$, there are $m$ functions $g^{(0)}, g^{(1)}, \cdots, g^{(m-1)}$ of $F_{1}^{\beta}$ such that, if $C_{2}^{(j)}, \cdots, C_{t+1}^{(j)}, D_{1}^{(j)}, \cdots, D_{t}^{(j)}(0 \leqq j \leqq m-1)$ are balls defined by

$$
\begin{array}{ll}
C_{i}^{(j)}=g^{(j)}\left(D_{i-1}^{(j)}\right) & (1<i \leqq t+1), \\
D_{i}^{(j)}=h_{i}\left(C_{1}, C_{2}^{(j)}, \cdots, C_{i}^{(j)}\right) & (1 \leqq i \leqq t),
\end{array}
$$

then $C_{t+1}^{(0)}, \cdots, C_{t+1}^{(m-1)}$ have pairwise disjoint interiors.

Under these assumptions, every $(\alpha, \beta)$-winning set $S \subset M$ has Hausdorff dimension at least

$$
\log m /|t \log \alpha \beta| \text {. }
$$

Corollary 1. Let $N(\beta)$ be such that every ball B contains a set of $N(\beta)$ balls of $B^{\beta}$ with pairwise disjoint interiors. Then every $(\alpha, \beta)$-winning set has Hausdorff dimension at least

$$
\log N(\beta) /|\log \alpha \beta| .
$$

For example on the real line one may put $N(\beta)=\left[\beta^{-1}\right]$. (That is, the integral part of $\beta^{-1}$.)

Proof. One may use the theorem with $t=1, m=N(\beta)$.

COROLlary 2. An $\alpha$-winning set in n-dimensional Euclidean space $E_{n}$ has Hausdorff dimension $n$. 
Proof. One has $N(\beta) \geqq c \beta^{-n}$ for some $c>0$. This gives the lower bound $(\log c+n|\log \beta|)(|\log \alpha|+|\log \beta|)^{-1}$, which tends to $n$ when $\beta$ tends to zero.

COROLlary 3. Let $1+\alpha \beta>2 \beta$. Then every $(\alpha, \beta)$-winning set in $E_{n}$ has positive Hausdorff dimension, and an $(\alpha, \beta)$-winning set in infinite-dimensional Hilbertspace has infinite Hausdorff dimension.

Proof. We first take the $n$-dimensional case. Write $\gamma=1+\alpha \beta-2 \beta>0$, and let the integer $t>1$ be so large that $(\alpha \beta)^{t}<\gamma / 3$.

Let $g^{i+}, g^{i-}(i=1, \cdots, n)$ be the functions of $F_{1}^{\beta}$ which assign to a ball $B$ of radius $\rho$ and center $\left(c_{1}, \cdots, c_{n}\right)$ the ball of radius $\beta \rho$ and center

$$
\left(c_{1}, \cdots, c_{i-1}, c_{i}+\rho(1-\beta), c_{i+1}, \cdots, c_{n}\right),\left(c_{1}, \cdots, c_{i-1}, c_{i}-\rho(1-\beta), c_{i+1}, \cdots, c_{n}\right),
$$

respectively.

Let $h_{1} \in F_{1}^{\alpha}, \cdots, h_{t} \in F_{t}^{\alpha}$, and let $C_{1}$ have center $c=\left(c_{1}, \cdots, c_{n}\right)$ and radius $\rho$. Let $1 \leqq j \leqq n$ and let $k$ denote + or - . Define $C_{2}^{j k}, \cdots, C_{t+1}^{j k} ; D_{1}^{j k}, \cdots, D_{t}^{j k}$ by

$$
\begin{array}{rlrl}
C_{i}^{j k} & =g^{j k}\left(D_{i-1}^{j k}\right) & & (2 \leqq i \leqq t+1), \\
D_{i}^{j k}=h_{i}\left(C_{1}, C_{2}^{j k}, \cdots, C_{i}^{j k}\right) & & (1 \leqq i \leqq t) .
\end{array}
$$

Denote the center of $C_{i}^{j k}$ by $\left(c_{i 1}^{j k}, \cdots, c_{i n}^{j k}\right)$. Then

$$
\begin{aligned}
& c_{2 j}^{j+} \geqq c_{j}+\rho(1-\beta-\beta(1-\alpha))=c_{j}+\rho \gamma>c_{j}, \\
& c_{t+1 j}^{j+} \geqq c_{j}+\rho \gamma .
\end{aligned}
$$

Hence $C_{t+1}^{j+}$, which has radius $\rho(\alpha \beta)^{t}<\rho \gamma / 3$, is contained in the halfplane $x_{j} \geqq c_{j}+2 \rho \gamma / 3$. Similarly, $C_{t+1}^{j-}$ is contained in $x_{j} \leqq c_{j}-2 \rho \gamma / 3$.

Let $l=l(\alpha, \beta)$ be an integer with $(l+1) \gamma^{2} / 9>1$. We claim that any ball $C_{t+1}^{j k}$ has nonempty intersection with at most $l$ of the balls $C_{t+1}^{j k}(j=1, \cdots, n ; k=+,-)$. To show this it suffices to see that $C_{t+1}^{1+}$ can intersect at most $l$ of these balls (including itself). By what has already been shown it cannot at the same time intersect $C_{t+1}^{j+}$ and $C_{t+1}^{j-}$. Thus it remains to show that $C_{t+1}^{1+}$ cannot intersect all the balls $C_{t+1}^{2+}, \cdots, C_{t+1}^{l+1+}$, say. If these intersections were nonempty, $c_{t+1}^{1+} \geqq c_{j}+\rho \gamma / 3$ $(j=2, \cdots, l+1)$, and the center of $C_{t+1}^{1+}$ would have distance from the center $c$ of $C_{1}$ at least $\rho \sqrt{ }\left(\gamma^{2}+l \gamma^{2} / 9\right)>\rho \sqrt{ }\left((l+1) \gamma^{2} / 9\right)>\rho$.

Thus there exist

$$
m=\max (2,2 n / l)
$$

of the balls $C_{t+1}^{j k}(j=1, \cdots, n ; k=+,-)$ which are pairwise disjoint. We can pick $m$ of the functions $g^{i k}$, say $g^{(0)}, \cdots, g^{(m-1)}$, which satisfy the conditions of the theorem. Therefore $S$ has Hausdorff dimension at least $\log m /|t \log \alpha \beta|>0$.

In the case of a Hilbertspace of infinite dimension the argument leading to (26) in the previous case shows that now one may take $m$ arbitrarily large. 
REMARK. The results of this section are in contrast to Folgerung 1 of Satz 3 of [8] where a different game is studied.

\section{Proof of Theorem 6.}

Lemma 20. Put $\omega=2 / \sqrt{ } 3-1$. Let $D, D_{1}, \cdots, D_{e}$ be balls in a Hilbertspace such that $\rho(D)<\omega \rho\left(D_{1}\right)=\cdots=\omega \rho\left(D_{e}\right)$. Let $D_{i}, D_{j}$ have disjoint interiors for $i \neq j$, and let $D, D_{i}$ have nonempty intersections for $i=1,2, \cdots, e$.

Then $e \leqq 2$.

Proof. We may assume $\rho\left(D_{1}\right)=\cdots=\rho\left(D_{e}\right)=1$. We have to show that the assumptions of the Lemma with $e=3$ lead to a contradiction.

Let $D$ have center $0, D_{i}$ center $x_{i}(i=1,2,3)$. Then $\left|x_{i}\right|<1+\omega,\left|x_{i}-x_{j}\right| \geqq 2$ for $i \neq j$. One obtains $\left|x_{i}\right|^{2}<(1+\omega)^{2}=4 / 3,4 \leqq\left|x_{i}-x_{j}\right|^{2}=\left|x_{i}\right|^{2}+\left|x_{j}\right|^{2}-2 x_{i} x_{j}$ (=inner product) $<8 / 3-2 x_{i} x_{j}$, hence $x_{i} x_{j}<-2 / 3 \quad(i \neq j)$. This gives $x_{1}\left(x_{2}+x_{3}\right)<-4 / 3$. On the other hand, $\left|x_{1}\left(x_{2}+x_{3}\right)\right|^{2} \leqq\left|x_{1}\right|^{2}\left|x_{2}+x_{3}\right|^{2}$ $<(4 / 3)\left(8 / 3+2 x_{2} x_{3}\right)<16 / 9$, which gives a contradiction.

Let $S$ be $(\alpha, \beta)$-winning, and let a winning strategy $f_{1}, f_{2}, \cdots$ be given. Call a sequence of balls $E_{1}, E_{2}, \cdots$ a $t-f_{1}, f_{2}, \cdots$-chain, if there is an $f_{1}, f_{2}$-chain $B_{1}, B_{2}, \cdots$ such that $E_{1}=B_{1}, E_{2}=B_{1+t}, E_{3}=B_{1+2 t}, \cdots$. Finite $t$-chains are defined similarly. In other words a $t$-chain consists of every $t$ th element of a chain.

LEMMA 21. Let all the hypotheses of the theorem be satisfied. Let $E_{1}, E_{2}, \cdots, E_{k}$ be a $t-f_{1}, f_{2}, \cdots$-chain. Then there are $m$ balls $E_{k+1}^{(0)}, \cdots, E_{k+1}^{(m-1)}$ with pairwise disjoint interiors such that each of the sequences $E_{1}, \cdots, E_{k}, E_{k+1}^{(j)}(j=0,1, \cdots, m-1)$ is a finite $t-f_{1}, f_{2}, \cdots$-chain.

Proof. Let $B_{1}, \cdots, B_{1+(k-1) t}$ be a $f_{1}, f_{2}, \cdots$-chain with $E_{1}=B_{1}, \cdots, E_{k}=B_{1+(k-1) t}$. Define $h_{i}\left(C_{1}, \cdots, C_{i}\right) \quad(i=1, \cdots, t)$ by $h_{i}\left(C_{1}, \cdots, C_{i}\right)=f_{i+(k-1) t}\left(B_{1}, \cdots, B_{(k-1) t}\right.$, $\left.C_{1}, C_{2}, \cdots, C_{i}\right)$. Put $C_{1}=B_{1+(k-1) t}=E_{k}$. Now let $g^{(0)}, \cdots, g^{(m-1)}$ be the functions of the theorem, and define $C_{2}^{(j)}, \cdots, C_{t+1}^{(j)}, D_{1}^{(j)}, \cdots, D_{t}^{(j)}(0 \leqq j \leqq m-1)$ by (24) and (25). Put $E_{k+1}^{(j)}=C_{t+1}^{(j)}$. Then obviously $E_{1}, \cdots, E_{k}, E_{k+1}^{(j)}$ is a $t-f_{1}, f_{2}, \cdots$ -chain for $0 \leqq j \leqq m-1$, and the $m$ balls $E_{k+1}^{(j)}$ have pairwise disjoint interiors.

LEMMA 22. Let all the hypotheses of the theorem be satisfied. There are balls $C_{1}\left(i_{1}\right), C_{2}\left(i_{1}, i_{2}\right), \cdots$, defined for digits $i_{j}=0,1, \cdots, m-1$, such that

$$
C_{1}\left(i_{1}\right), C_{2}\left(i_{1}, i_{2}\right), C_{3}\left(i_{1}, i_{2}, i_{3}\right), \cdots
$$

is a $t-f_{1}, f_{2}, \cdots$-chain for every sequence of digits $i_{1}, i_{2}, \cdots$, and where for given $k$ the $m^{k}$ balls $C_{k}\left(i_{1}, \cdots, i_{k}\right)$ have pairwise disjoint interiors and radius $(\alpha \beta)^{k t}$.

Proof. Let $C_{1}\left(i_{1}\right)$ be any $m$ disjoint balls of radius $(\alpha \beta)^{t}$. The construction of $C_{2}\left(i_{1}, i_{2}\right), C_{3}\left(i_{1}, i_{2}, i_{3}\right), \cdots$ is by induction, using the previous lemma. 
Proof of Theorem 6. Given a sequence of digits $i_{1}, i_{2}, \cdots$ there is a unique point $x=x\left(i_{1}, i_{2}, \cdots\right)$ contained in all the balls $C_{k}\left(i_{1}, \cdots, i_{k}\right), k=1,2, \cdots$ of Lemma 22 . Obviously $x \in S$. The set of all points $x$ so obtained will be denoted by $S^{*}$.

Define a possibly many-valued function $f$ from $S^{*}$ onto the unit-interval $U: 0 \leqq y \leqq 1$, as follows. Given $x \in S^{*}$, let $f(x)$ consist of all numbers $y=0, i_{1} i_{2} \cdots$ (written in scale $m$ ) such that $x=x\left(i_{1}, i_{2}, \cdots\right)$. For a set $T \subset S^{*}$ let $f(T)$ be the union of all sets $f(x)$ where $x \in T$. For a general set $R$ define $f(R)=f\left(R \cap S^{*}\right)$. Now if balls $B_{l}(l=1,2, \cdots)$ cover $S$, the sets $B_{l} \cap S^{*}$ cover $S^{*}$, and the sets $f\left(B_{l}\right)=f\left(B_{l} \cap S^{*}\right)$ cover $U$. Hence the exterior Lebesgue measures $\bar{\mu}$ of $f\left(B_{l}\right)$ satisfy

$$
\sum_{l=1}^{\infty} \bar{\mu}\left(f\left(B_{l}\right)\right) \geqq 1
$$

Let $B$ have radius $\rho$, and put

$$
j=\left[\log \left(2 \rho \omega^{-1}\right) /(t \log \alpha \beta)\right] .
$$

For small $\rho, j$ is positive, and

$$
\rho<\omega(\alpha \beta)^{t j}
$$

Hence by Lemma 20,B has nonempty intersection with at most two of the balls $C_{j}\left(i_{1}, \cdots, i_{j}\right)$, say with $C_{j}\left(i_{1}(1), \cdots, i_{j}(1)\right)$ and $C_{j}\left(i_{1}(2), \cdots, i_{j}(2)\right) . f(B)$ contains only numbers whose first $j$ digits are either $i_{1}(1), \cdots, i_{j}(1)$ or $i_{1}(2), \cdots, i_{j}(2)$. Thus $f(B)$ is contained in two intervals of length $m^{-j}$, and $\bar{\mu}(f(B)) \leqq 2 m^{-j}$.

Now suppose the balls $B_{1}, B_{2}, \cdots$ of radius $\rho_{1}, \rho_{2}, \cdots$ cover $S$. By (27),

$$
1 \leqq 2 \sum_{t=1}^{\infty} m^{-j_{t}}
$$

where $j_{l}$ is defined by a formula like (28). This implies

$$
1 \leqq 2 m \sum_{l=1}^{\infty}\left(2 \omega^{-1} \rho_{l}\right)^{\log m /|t \log \alpha \beta|}
$$

We obtain $\{S\}^{\alpha}>0$ with $\alpha=\log m /|t \log \alpha \beta|$, and the theorem is proved.

LEMMA 23. Let $2 \beta<1+\alpha \beta$ and let $S$ be $(a, \beta)$-winning in a Hilbertspace $M$ of positive dimension. The intersection of $S$ with any ball contains continuummany points.

Proof. The proof of Corollary 3 of Theorem 6 shows that this theorem is applicable with some $m>11$ and with $C_{t+1}^{(0)}, \cdots, C_{t+1}^{(m-1)}$ disjoint. Under this assumption the $m^{j}$ balls $C_{j}\left(i_{1}, \cdots, i_{j}\right)$ of Lemma 22 will be pairwise disjoint. One may also require that all the balls $C_{j}\left(i_{1}, \cdots, i_{j}\right), j=1,2, \cdots$, be contained in an arbitrary fixed ball $B$ if one drops the inessential requirement on the radii 
of these balls. The points $x\left(i_{1}, i_{2}, \cdots\right)$ will now be continuum-many distinct points of $S \cap B$.

\section{Positional winning strategies.}

TheOREM 7. Let $S \subset M$ be an (F, (5)-winning set. Then there exists a positional $(\mathfrak{F},(\mathfrak{5} ; S)$-winning strategy.

Proof. Introduce a well-ordering $\prec$ into the set $\Omega$. Let $f_{1}, f_{2}, \cdots$ be an $(\mathfrak{F},(\mathfrak{5} ; S)$ winning strategy. We are going to define $f \in F_{1}$ as follows.

Let $B \in \Omega$. If $B$ does not occur in any $f_{1}, f_{2}, \cdots$-chain, define $f(B)$ arbitrarily. Now assume $B$ does occur in $f_{1}, f_{2}, \cdots$-chains

$$
B_{1}, B_{2}, \cdots, B_{k}=B \text {. }
$$

Of all the $B_{1}$ which occur in such chains, there is one which is smallest with regard to $\prec$. Denote it by $B_{1}(B)$. There are chains

$$
B_{1}(B), B_{2}, \cdots, B_{h}=B \text {. }
$$

Of all the $B_{2}$ which occur in such chains, there is a smallest one, $B_{2}(B)$, and so on. Now either

(a) there is a $k$ with $B_{k}(B)=B$. Then

$$
B_{1}(B), \cdots, B_{k}(B)=B
$$

is an $f_{1}, f_{2}, \cdots$-chain.

(aa) There is no $f_{1}, f_{2}$-chain $C_{1}, C_{2}, \cdots$ where each $C_{i}$ is one of the elements $B_{1}(B), \cdots, B_{k}(B)$. If for every $m$ there were a finite $f_{1}, f_{2}, \cdots$-chain $C_{1}, \cdots, C_{m}$ with this property, then because of Lemma 1 there would also be an infinite such chain $C_{1}, C_{2}, \cdots$. Let $B_{1}(B), \cdots, B_{k}(B), C_{1}, \cdots, C_{i_{0}}$ be an $f_{1}, f_{2}$-chain with each $C_{i}$ among the $B_{1}(B), \cdots, B_{k}(B)$, such that there is no longer such chain. Set

$$
f(B)=f_{k+i_{0}}\left(B_{1}(B), \cdots, B_{k}(B), C_{1}, \cdots, C_{i_{9}}\right) .
$$

If $B^{\prime} \in \mathfrak{G}(f(B)), B^{\prime}$ differs from $B_{1}(B), \cdots, B_{k}(B)$.

(ab) There is an $f_{1}, f_{2}, \cdots$-chain $C_{1}, C_{2}, \cdots$ with each $C_{i}$ among $B_{1}(B), \cdots, B_{k}(B)$. In this case $\bigcap_{i=1}^{k} \alpha\left(B_{i}(B)\right) \subset \bigcap_{n} \alpha\left(C_{n}\right) \subset S$. Hence $\alpha(B)=\alpha\left(B_{k}(B)\right) \subset S$, and $f(B)$ can be any element of $\mathfrak{F}(B)$.

(b) There is no $k$ having $B_{k}(B)=B$. Then $B_{1}(B), B_{2}(B), \cdots$ is an $f_{1}, f_{2}$-chain, and $\alpha(B) \subset \bigcap \alpha\left(B_{n}\right) \subset S$. Again $f(B)$ can be arbitrary in $\mathfrak{F}(B)$.

We are going to show that the functions $\bar{f}_{n}\left(B_{1}, \cdots, B_{n}\right)$ defined by $\bar{f}_{n}\left(B_{1}, \cdots, B_{n}\right)$ $=f\left(B_{n}\right)(n=1,2, \cdots)$ are a winning strategy. Let $B_{1} \in \Omega^{\prime}$,

$$
\begin{array}{ll}
B_{n} \in \mathfrak{G}\left(W_{n-1}\right) & (n=2,3, \cdots), \\
W_{n}=f\left(B_{n}\right) & (n=1,2, \cdots) .
\end{array}
$$


If for some $B_{n}$, case (ab) or (b) happens, $\alpha\left(B_{n}\right) \subset S$, and we are through. Thus for every $B_{n}$, assume (aa) holds.

$$
B_{1}\left(B_{n}\right), \cdots, B_{k}\left(B_{n}\right), C_{1}, \cdots, C_{i_{0}}, B_{n+1}
$$

is an $f_{1}, f_{2}, \cdots$-chain, and $B_{n+1}$ differs from $B_{1}\left(B_{n}\right), \cdots, B_{k}\left(B_{n}\right)$.

$$
B_{1}\left(B_{n+1}\right) \prec B_{1}\left(B_{n}\right) \quad(n=1,2, \cdots) .
$$

There is an $i_{1}$ where $B_{1}\left(B_{i_{1}}\right)$ is smallest with regard to $\prec$. By $(29), B_{1}\left(B_{i}\right)$ $=B_{1}\left(B_{i_{1}}\right)=\overline{B_{1}}$, say, if $i \geqq i_{1}$. Now for $i>i_{1}, B_{i}$ differs from $B_{1}\left(B_{i-1}\right)=\overline{B_{1}}$ $=B_{1}\left(B_{i}\right)$. Thus $B_{2}\left(B_{i}\right)$ is defined. There is an $i_{2}>i_{1}$ such that $B_{2}\left(B_{i_{2}}\right)=B_{2}\left(B_{i}\right)$ for $i \geqq i_{2}$. In this fashion one finds $i_{1}<i_{2}<\cdots$ such that $B_{t}\left(B_{i}\right)$ is defined for $i>i_{t-1}$ and $B_{t}\left(B_{i}\right)=B_{t}\left(B_{i_{t}}\right)=\overline{B_{t}}$ for $i \geqq i_{t} . \overline{B_{1}}, \bar{B}_{2}, \cdots$ is an $f_{1}, f_{2}, \cdots$-chain by Lemma 1.

$$
\bigcap_{i=1}^{\infty} \alpha\left(B_{i}\right) \subset \bigcap_{i=1}^{\infty} \alpha \overline{\left(B_{i}\right)} \subset S
$$

gives the desired conclusion.

\section{REFERENCES}

1. H. Davenport, A note on Diophantine approximation. II, Mathematika 2 (1964), 50-58.

2. Morton Davis, Infinite games of perfect information, Annals of Mathematics Studies No. 52, Princeton Univ. Press, Princeton, N. J., 1964; pp. 85-101.

3. D. Gale and F. M. Stewart, Infinite games with perfect information, Annals of Mathematics Studies No. 28, Princeton Univ. Press, Princeton, N. J., 1953; pp. 245-266.

4. A. Ya. Khintchine, Continued fractions, English transl. by P. Wynn, Noordhoff, Groningen, 1963.

5. J. Mycielski, S. Swierczkowski and A. Zieba, On infinite positional games, Bull. Acad. Polon. Sci. Cl. III 4 (1956), 485-488.

6. J. C. Oxtoby, The Banach-Mazur game and Banach Category Theorem, Annals of Mathematics Studies No. 39, Princeton Univ. Press, Princeton, N. J., 1957; pp. 159-163.

7. W. M. Schmidt, On badly approximable numbers, Mathematika 12 (1965), 10-20.

8. B. Volkmann, Gewinnmengen, Arch. Math. 10 (1959), 235-240.

\section{UNIVERSITY OF COLORADO,}

$$
\text { BOULDER, CoLORADO }
$$

